\title{
Randomization for Multi-agent Constraint Optimization
}

\author{
Student: Quang Huy NGUYEN \\ Supervisor: Prof. Boi V. FALTINGS \\ Artificial Intelligence Laboratory (LIA) \\ Swiss Federal Institute of Technology Lausanne (EPFL), Switzerland \\ \{quanghuy.nguyen, boi.faltings\}@epfl.ch
}

In a constraint optimization problem for multiple agents, the agents have conflicting preferences in the final solution and the goal is to find an optimal assignment that maximizes total utilities of all agents. Two major challenges when solving constraint optimization problems for multiple agents are the complexity of finding optimal solution and the incentive compatibility for participating agents. First, computing the optimal solution for large optimization problems are computationally infeasible and it can only be solved approximately by local search algorithms. Second, ensuring honest elicitation among self-interested agents is computationally expensive. It has been shown that the only known mechanism that guarantees truthfulness among agents requires computing optimal solutions, and sub-optimal solutions for such a mechanism will break the incentive compatibility ([2]).

The long-term goal of our research is to solve these two challenges by using randomization in local search algorithms to find near-optimal solutions while ensuring incentive compatibility for bounded-rational agents. Our work is based on the observation that in real-world settings, the potential for manipulation is limited by uncertainty and risk. This uncertainty makes it difficult for a manipulator to predict the consequences of his manipulation and thus makes attempts at manipulating it uninteresting.

In this paper we discuss a general randomization technique, called random subset optimization, for escaping from local minima in local search algorithms. In each local choice step, the local search procedure will randomly choose a part of the optimization function, and optimize for this part only. It turns out that this results in a more focussed optimization strategy and is shown to be especially effective in some hard optimization problems. We show that the uncertainty of our randomization algorithms can make the agents' manipulation hard, thus prevent bounded rational agents from manipulating such algorithms. We propose a new hard-to-manipulate local search algorithm using our randomization scheme ([1]). Experiments on randomly generated problems and benchmarks from DIMACS show that our technique is very promising: it outperforms existing local search algorithms both in speed of convergence and solution quality on both decomposable and non-decomposable problems.

\section{References}

1. B. Faltings and Q.-H. Nguyen. Multi-agent coordination using local search. In Proceedings of IJCAI 05, 2005.

2. Noam Nisan and Amir Ronen. Computationally feasible VCG mechanisms. In Proceedings of the 2nd ACM conference on Electronic commerce, pages 242-252. ACM Press, 2000. 